\title{
ESTUDIO TAXONÓMICO Y CONSERVACIÓN DE PTERIDOFITOS DE LOS VALLES COSTEROS DE TACNA
}

Juan Franco León'

Liduvina Sulca Quispe ${ }^{\text {I }}$

\author{
$R E S U M E N$
}

El presente trabajo reporta el estudio de pteridofitos de los valles costeros Locumba, Sama y Caplina del departamento de Tacna, realizado entre julio de 1995 y enero de 1996.

El objetivo principal fue establecer la taxonomía y conservación de los pteridofitos que se encuentran en el área estudiado. El muestreo se realizó en la ribera de los ríos, borde de acequias y áreas próximas y para la clasificación de los especímenes se empleó el método descriptivo relacionado con la observación de las estructuras macro y microscópicas de las muestras. Los datos obtenidos se confrontaron con claves de identificación, se tomaron en cuenta algunas características ecológicas del medio.

Como resultado del estudio realizado se ha logrado determinar las siguientes especies: Equisetum giganteum (Equisetaceae) Pityrogramma trifoliata (Pteridaceae), Thelypteris rufa (Thelypteridaceae) y Nephrolepis sp (Davalliaceae), presentándose como especie predominante $\boldsymbol{E}$. giganteum, el cual tiene importancia como recurso natural medicinal en la zona.

\section{A B S TRA C T}

The present work reports the study of pteridofitos of the coastal valleys of Locumba, Sama and Caplina in the department of Tacna, carried out from July 1995 to January 1996.

The main objective was to establish the taxonomy and conservation of the pteridofitos that are in the studied area. The sampling was carried out on the banks of rivers, the border of canals, and the inmediately surrounding area and for the classification of the specimns the descriptive method related to the observation of the macro and micro structures of the samples was used. The obtained data was classified with identification keys; some ecological characteristics of the media were taken into account.

As a result of the study, it has been possible to determine the following species: Equisetum giganteum (Equisetaceae) Pityrogramma trifoliata (Pteridaceae), Thelypteris rufa (Thelypteridaceae) and Nephrolepis sp (Davalliaceae), with the predominant species being $\boldsymbol{E}$. giganteum, which has importance as a natural medicinal resource in the area. 


\section{INTRODUCCIÓN}

\section{HEH JUOIECA GENTPAL}

Los Pteridofitos, llamados plantas vasculares inferiores, son conocidos vulgarmente como "helechos". A diferencia de los hongos y briofitos son plantas diferenciadas en una raiz, tallo y hojas. Se hallan difundidas por todo el globo y en las aguas dulces continentales; las especies terrícolas se encuentran de preferencia en las estaciones dotadas de cierto grado de humedad, por lo menos temporalmente ya que parte de su ciclo biológico aunque breve pero de gran importancia requiere de manera absoluta la presencia de agua.

Para el Perú se reportan más de 1000 especies (León, 1993), (Tryon, et al, 1994) distribuidas en ambientes terrestres húmedos o dulceacuícolas de la costa, sierra y selva; su difusión puede alcanzar altitudes de 4500 m.s.n.m. (Orellana, 1992). Se han descrito una diversidad de especies en cuanto a número, forma y tamaño; es posible encontrar especies pequeñas como Azolla filiculoides, "lentejita de agua" y especies tan grandes como los helechos arborescentes del género $C$ yathea propios de nuestra amazonía.

Desde los estudios realizados por Weberbauer (1901) hasta la fecha numerosos investigadores han reportado diversas especies, muchas de ellas nuevas para la ciencia.

La creciente preocupación de algunos estudiosos ha permitido ampliar el conocimiento sobre los pteridofitos del Perú; las investigaciones se han realizado en el Nor-Oeste peruano (Sagástegui, 1989), en el centro (Orellana, 1994), en el sur (Cáceres, 1994) entre otros lugares.

Otros investigadores incidieron en el estudio de las propiedades medicinales de los helechos, contribuyendo así al conocimiento de la flora medicinal. Sin embargo, existen zonas del país en donde los estudios botánicos son escasos, probablemente por la falta de interés de aquellos lugares.

La costa sur presenta las características de un desierto por la ausencia de lluvias, pero ello no impide el desarrollo de especies vegetales como los pteridofitos, los cuales se encuentran en los valles costeros junto al borde de los ríos y acequias dotadas de agua permanente en donde encuentran el ambiente propicio para su desarrollo.

Para esta región se reportan como especies de mayor difusión: Equisetum giganteum,
Pityrogramma trifoliata y Polypodium en otras especies. (Cáceres, 1994) asimismo son consideradas como parte de la flora regional.

Hasta la fecha no existen reportes sobre los pteridofitos de los valles costeros del departamento de Tacna y por lo tanto su conocimiento es escaso. Tomando en cuenta lo expuesto anteriormente el presente trabajo de investigación se planteó como objetivo el estudio y conservación de los helechos de la zona mencionada y de esta manera incrementar el conocimiento de los pteridofitos de nuestro país.

\section{MATERIAL Y MÉTODOS}

Se determinó como estaciones de muestreo diferentes localidades de los valles de Locumba, Sama y Caplina, en los cuales se realizó la recolección del material biológico entre los meses de julio a diciembre de 1995. El muestreo se efectuó en lugares húmedos, especialmente en la ribera de los ríos, borde de acequias y lugares próximos.

La recolección de un helecho se hizo cogiendo la planta integramente incluyendo el rizoma y en lo posible con los frondes debidamente fructificados. Las muestras colectadas (frondes) se procedieron a herborizar, haciéndose el prensado de las mismas; una vez procesadas y secas, los ejemplares fueron montados en cartulinas para su posterior identificación. La colecta de equisetos consistió en coger los ejemplares que reunian todas las características morfológicas que permitan su identificación; se tomaron porciones representativas del tallo y al igual que los rizomas fueroncolocados porseparadoenfrascosconteniendo formol al $5 \%$ para la conservación de la muestra. Las cartulinas montadas y los frascos conteniendo las muestras fueron adjuntados de una boleta indicando el lugar de recolección, hábitat, tamaño del ejemplar y la altitud en el cual se les halló.

El material colectado y debidamente procesado fue trasladado al Laboratorio de Botánica de la Universidad Nacional "Jorge Basadre Grohman" de Tacna en donde fueron identificados.

Para la determinación de los especímenes se empleó el método descriptivo relacionado con la observación macroscópica de los órganos, haciéndose uso del estereoscopio y microscopio en este último caso. Las características tomadas en cuenta fueron:

- Forma y tamaño de la fronda.

- Forma y posición de los soros o estróbilos.

- Forma de las esporas. 
- Presencia y forma del indusio

- Presencia de pelos y escamas, etc.

Los datos obtenidos durante las observaciones fueron comparados con la clave de identificación de Fernández (1987). Se tomaron fotografías del material estudiado.

Muestras y fotografías del material procesado fueron enviados al Departamento de Geografía de la Universidad de Maryland, Baltimore-USA, para la respectiva confirmación de las especies.

\section{RESULTADOS}

El estudio taxonómico realizado en los valles costeros del departamento de Tacna nos permite clasificar a los pteridofitos en dos órdenes, cuatro familias y cuatro géneros cada uno de ellos con una especie representativa.

La sistemática trazada para la ubicación de las especies en la División Pteridophyta es como se da a continuación:

\section{DIVISIÓN PTERIDOPHYTA}

Clase : Sphennopsida

Orden : Equisetales

Familia : Equisetaceae

Especie : Equisetum giganteum L. (Milde)

Clase : Filicopsida (Pteropsida)

Subclase: Polypodidae

Orden : Polypodiales

Familia : Pteridaceae

Especie : Pityrogramma trifoliata (L)

$$
\text { R. Tryon }
$$

Familia : Thelypteridaceae

Especie : Thelypteris rufa (Poiret) A.R. Smith

Familia : Davalliaceae

Especie : Nephrolepissp.

\section{DESCRIPCIONES DE LAS ESPECIES IDENTIFICADAS}

Aspecto relativos a las especies identificadas se describen a continuación resaltando las características morfológicas tomando en cuenta las observaciones macroscópicas y microscópicas realizados durante la identificación y las que se obtuvieron de la clave y la bibliografía consultada. Finalmente, se consignan datos referentes al hábitat y distribución en el área estudiada.

a) Equisetum giganteum(Fig. 1). Plantaperenne rizomatosa. Rizoma subterráneo tuberiforme alargado de color negro-violaceo, fistuloso sin sílice no carenado, nudoso. Presenta tallo aéreo vertical, cilíndrico, articulado con internodios manifiestos, verde oscuro, nudoso y áspero con 20 a 40 carenas con incrustaciones de sílice y Valéculas angostas; llega a medir hasta $6 \mathrm{~m}$. de alto.

Del tallo emergen las ramas, también articuladas en segmentos y se disponen en verticilos sobre los nudos; la epidermis es rugosa en las carenas y en las valéculas. Espiga fructífera estrobiliforme cilíndrica, apiculada y sésil ubicado en el extremo de las ramas. Los esporófilos tienen forma de clavo de amplia cabeza, peltado poligonal; en su cara inferior llevan de 6 a 8 esporangios sésiles oblongo-sacciformes que se abren interiormente. Las esporas esféricas café-pardas muy higroscópicas y con presencia de elaterios hialinos.

Fig. 1. Equisetum giganteum.

1. Fragmento del tallo; 2. Estróbilo; 3. Espora; 4. Esporófilo; 5. Rizoma.

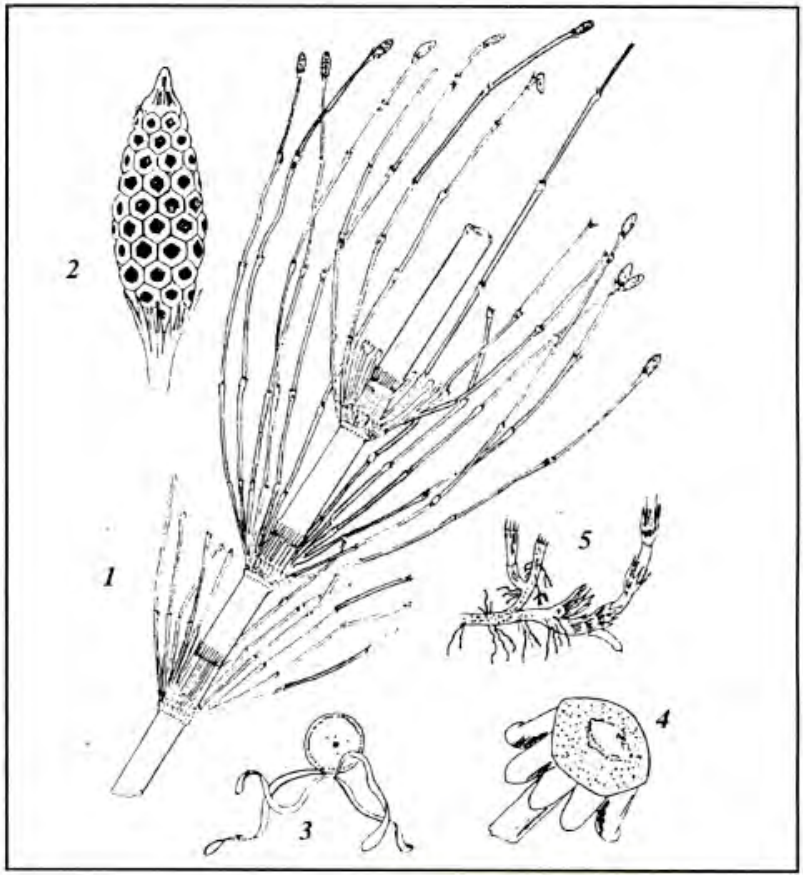


Es una especie propia de América. Actualmente se distribuye en las regiones templadas y cálidas; prefiere lugares húmedos muy bien abrigados contra el viento. En los valles costeros de Tacna esta especie habita exclusivamente en la ribera de los ríos Locumba, Sama y Caplina, borde de acequias y linderos de los campos de cultivo dotados de agua permanente. Su distribución está desde los $200 \mathrm{~m}$. de altitud (parte baja del valle de Locumba) hasta más allá de los $1200 \mathrm{~m}$. de altitud (Anexos de Calientes y Tocuco, en la parte alta del valle del Caplina). Su estado de conservación es adecuado para ser utilizado como recurso natural medicinal.

b) Pityrogramma trifoliata (Fig. 2). Helecho herbáceo de frondas compuestas desarrollada, pinnadas no muy rígidas, por lo común de 1 a $1.5 \mathrm{~m}$. de longitud. Pinnas bifoliadas y característicamente trifoliadas (ternadas), pequeñas y alargadas. Rizoma erecto con tricomas o escamas. Esporangios agrupados en líneas sorales o dispuestos en serie de tal manera que cubren casi la totalidad del envé del foliolo excepto la costa y los bordes periféricos; acompañan formaciones seríceas y escamas blancoamarillentas. Las esporas son esféricas de color café a pardo oscuro. El indusio está ausente y el raquis es glabro.

Fig. 2. Pityrogramma trifoliata.

1. Porción inferior de la fronda; 2. Porción superior de la fronda; 3.Pinna mostrando los soros; 4. Esporas.

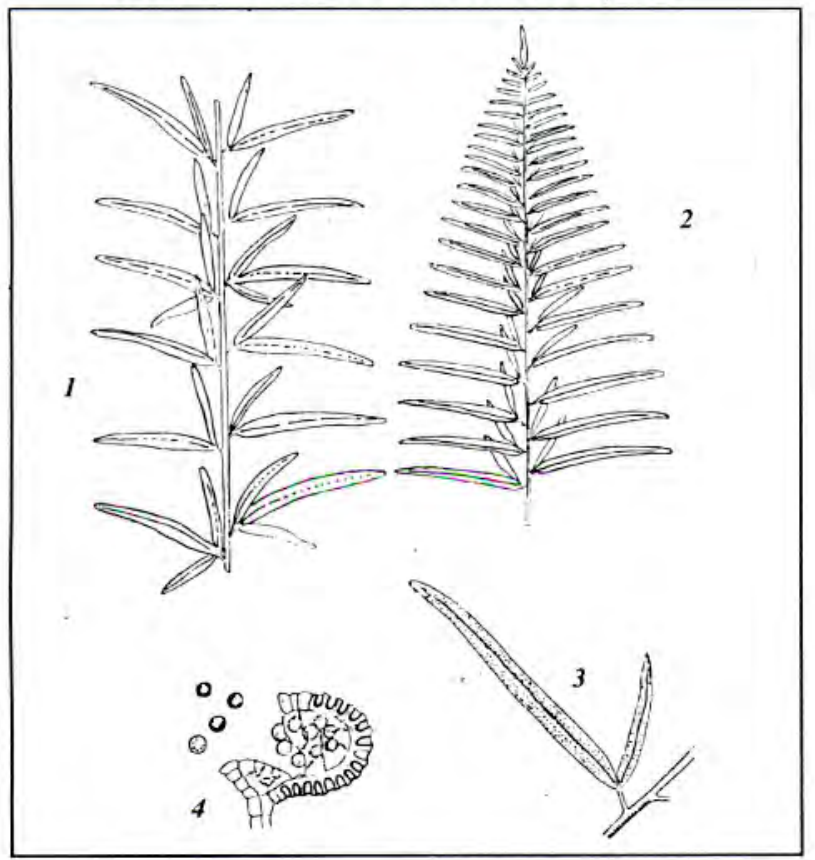

Su distribución es amplia sobre todo en la costa peruana. Habita en ambientes húmedos, en laderas rocosas o a lo largo de los canales del agua. En la zona estudiada se distribuye principalmente en el valle del Caplina hasta una altitud por encima de los 1200 m.s.n.m. exclusivamente en los bordes de las acequias. Generalmente se presenta en forma aislada y escasa.

c) Thelypteris rufa (Fig. 3). Helecho de frondas amplias y rígidas por lo común dispuestas en forma de corona; alcanzan hasta 1,5 m. de longitud. Las frondas llevan de 40 a 60 pares de pinnas opuestas o alternas, cada una de ellas de 9 a $13 \mathrm{~cm}$. de longitud y con 20 a 40 pares de pinnulas comúnmente opuestas y oblongas. Peciolo largo y delgado, glabro, verdeamarillo en las hojas jóvenes y café-marrón de diversas tonalidades en las hojas adultas. Soros circulares, con indusio, en número de 8 a 12 , situados en el envés de las pinnulas no muy próximos al margen. Las esporas son reniformes.

El rizoma erecto o alargado de color café oscuro. Realizando un corte transversal del rizoma se observan haces vasculares en número de 5 .

Se distribuye en áreas muy húmedas y sombrías. En Tacna su hábitat está limitado sobre todo a los bordes de las acequias ubicadas en la parte alta del valle del Caplina, sobre los $1000 \mathrm{~m}$. de altitud. Se presenta escasamente y se ve amenazado por la masiva destrucción de los campos.

Fig. 3. Thelypteris rufa.

1. Esporofito (fronda y rizoma); 2. Porción de la fronda; 3.Pinnula mostrando los soros; 4. Sección transversal del rizoma: 5. Esporas.

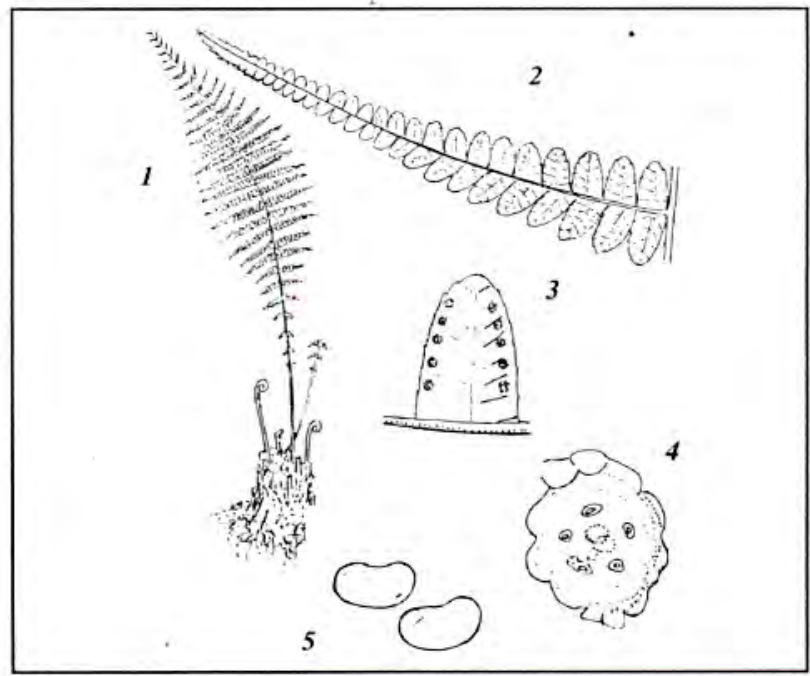


d) Nephrolepis sp. (Fig. 4). Caracterizado por presentar frondas erectas y raquis no glabro, Pinnas rígidas lanceoladas por lo común de 4 a $5 \mathrm{~cm}$. de longitud ligeramente recurvadas y con los bordes aserrados hacia las puntas, las cuales son agudas. A

Fig. 4: Nephrolepis sp.

1. Porción superior de la fronda: 2. Porción inferior de la fronda; 3. Pinnas con soros; 4. Sección vertical de un soro: 5. Indusio; vista superior:

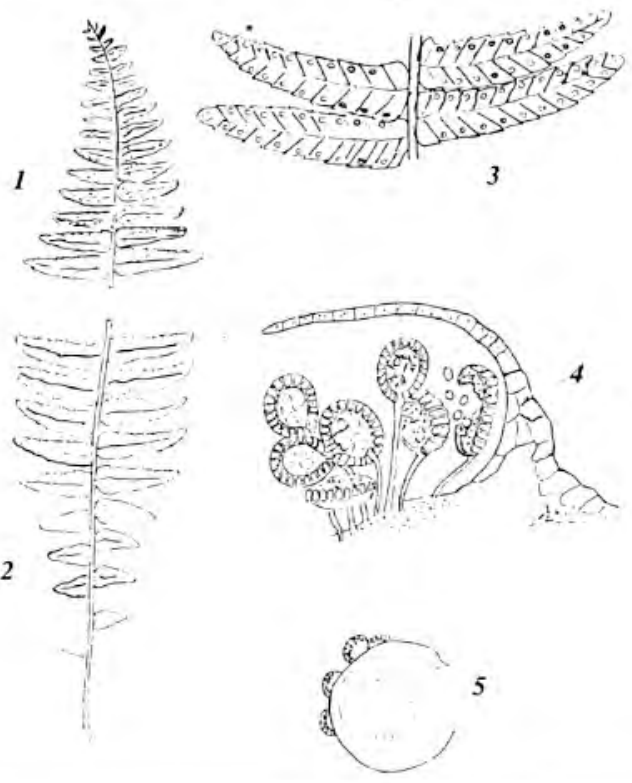

\section{REFERENCIAS BIBLIOGRÁFICAS}

ALDAVE A.; MOSTACERO L. 1998. Botánica Farma-céutica. Edit. Libertad, Perú.

CÁCERES, F. 1994. Estudio de algunas pteridophytas de la Provincia de Camaná (Dpto. de Arequipa) - Libro resumen del VI Congreso Nacional de Botánica, UNSAAC. Cusco-Perú.

CRÓNQUIST, A. 1990. Introducción a la Botánica, Edit. Continental S.A. México.

FERNÁNDEZ, H. 1995. Manual práctico de Botánica Criptogámica, Edit. Universidad Nacional de Trujillo-Perú.

FLORES, J.; VELARDE, L. 1995. Helechos medicinales de la Familia Polypodiaceae del Santuario histórico de Machupicchu, Libro resumen del VICongreso Nacional de Botánica. UNSAAC. CuscoPerú.

GOLA, G., OTROS 1965. Tratado de Botánica, 3ro. Edic., Edit. Labor S.A. Barcelona.

LEÓN, B. 1995. Actualización de los nombres de pteridofitos en la Obra Weberbauer (1945). Mueseo de la Historia Natural. LimaPerú. su vez la pinnas son alternas y glabras. Los soros son circulares con presencia de indusio circularcordiforme, situados en el extremo de las nervaduras secundarias. Su tamaño por lo común de $60 \mathrm{~cm}$. a 1 m. de longitud.

Es una especie introducida en Tacna, su difusión es mayor en la ciudad y escasamente en el campo en donde prefiere los bordes de acequias ubicadas en los alrededores de la Ciudad.

\section{CONCLUSIONES}

- Hasta el momento se ha identificado 4 Familias cada una de ellas con una especie representativa.

- La especie mejor representada y ampliamente distribuida en la zona es E. giganteum.

- Se observó que el predominio de los helechos polipodiaceos se encuentra junto a las acequias y canales de agua, siendo escasos en la ribera de los ríos.

- Nephrolepis sp. tiene presencia limitada y escasa en los alrededores de la ciudad de Tacna.

- Hasta el momento existe un equilibrio en el uso de E. giganteum como recurso natural medicinal.

ORELLANA, R. 1992. Revisión de los Helechos (Pteridophyta) del Departamento de Junín(I parte)-LibroresumendelVICongreso Nacional de Botánica. UNSAAC. Cusco-Perú.

SAGÁSTEGUI, A. 1973. Manual de malesas de la Costa Nor-Peruana. Edit. Taller Gráfico de la Unviersidad Nacional de Trujillo. Perú.

SAGÁSTEGUI, A. 1989. Vegetación y Flora de la Provincia de Contumaza. Edit. Libertad, Trujillo-Perú.

TRYON, R.M. Y R.G. STOLZA 1989. Peridophyta of Perú, Part. IV Pteridaceae - Dennstaedtiaceae. Fieldiana Bot. N.S. 22: 1-128.

URRUNAGA, R.; HOLGADO, M. Cryptogamas útiles en la Región Inka. Libro resumen del VI Congreso de Botánica. UNSAAC, Cusco-Perú.

WEBERBAUER, A. 1945. El Mundo vegetal de los Andes Peruanos. Ministerio de Agricultura, Lima.

WILSON, C.; LOOMIS, W. 1980. Botánica, 4ta. edic. Edit. HispanoAmericana CE de C.V. México. 